\title{
Risk of QT Prolongation through Drug-drug Interactions between Hydroxychloroquine and Concomitant Drugs Prescribed in Real-world Practice
}

\section{Byung Jin Choi}

Ajou university, School of medicine

\section{Yeryung Koo}

Ajou university, School of medicine

Tae Young Kim

Ajou university, School of medicine

Wou Young Chung

Ajou university, School of medicine

Yun Jung Jung

Ajou university, School of medicine

Ji Eun Park

Ajou university, School of medicine

Hong-Seok Lim

Ajou university, School of medicine

Bumhee Park ( $\sim$ bhpark@ajou.ac.kr)

Ajou university, School of medicine

Dukyong Yoon ( $\nabla$ d.yoon.ajou@gmail.com )

Ajou university, School of medicine

\section{Research Article}

Keywords: QT interval electrocardiography, QT prolongation, Drug Interactions, COVID-19, Hydroxychloroquine, Polypharmacy

Posted Date: September 22nd, 2020

DOI: https://doi.org/10.21203/rs.3.rs-79572/v1

License: (9) (1) This work is licensed under a Creative Commons Attribution 4.0 International License. Read Full License 


\section{Abstract}

Background: Hydroxychloroquine has recently received attention as a treatment for COVID-19. However, hydroxychloroquine may prolong the QTc interval, thus increasing the risk of life-threatening arrhythmia. Many patients with COVID-19 have comorbidities, necessitating the use of several drugs simultaneously with hydroxychloroquine. However, the risk of QT prolongation due to drug-drug interactions (DDIs) between hydroxychloroquine and these co-medications has not been identified. Therefore, it is necessary to investigate the risk of QT interval prolongation due to DDIs between hydroxychloroquine and frequently used concurrent drugs.

Methods and Results: Using 447,632 patients and 1,040,752 electrocardiograms, we investigated the risk of QT prolongation due to DDIs between hydroxychloroquine and 118 concurrent drugs frequently used in real-world practice. In the analysis, we observed that 11 drugs (trimebutine, tacrolimus, tramadol, rosuvastatin, ciclosporin, sulfasalazine, rofecoxib, diltiazem, piperacillin/tazobactam, and isoniazid) show DDIs with hydroxychloroquine in the direction of QT prolongation.

Conclusions: We found 11 drugs that show significant $(p<0.05)$ DDIs with hydroxychloroquine, thereby increasing the risk of QT prolongation in patients. It is necessary to consider prescribing alternative drugs that have less DDI when these drugs are concurrently administered with hydroxychloroquine. Further investigation is needed to assess more profoundly the risk of QT prolongation due to DDI with hydroxychloroquine of each drug that we found in this analysis.

\section{Introduction}

The COVID-19 pandemic has caused more than 14 million cases of the disease worldwide as of July 22, $2020,{ }^{1}$ and no drug has been proven to target this virus to date. Meanwhile, hydroxychloroquine, a conventional antimalarial treatment, has been used to treat COVID-19 in off-label practice. ${ }^{2}$ The efficacy and acceptable safety of hydroxychloroquine have been reported in multicenter clinical trials conducted in China. ${ }^{3}$

However, several controversies exist, especially regarding the cardiotoxic side effects of hydroxychloroquine, because previous studies have provided evidence that the use of hydroxychloroquine increases the risk of sudden cardiac death due to QT prolongation. ${ }^{4}$ Furthermore, when hydroxychloroquine is administered together with other drugs, it can exacerbate the risk of QT prolongation due to drug-drug interactions (DDIs). ${ }^{5}$ Although some studies have reported that hydroxychloroquine is more effective in treating COVID-19 when administered along with azithromycin, 6 ,

7 others claim that the combination of hydroxychloroquine and azithromycin does not show any clinical benefit or antiviral clearance, ${ }^{8}$ and can substantially increase the risk of QT prolongation. ${ }^{9,} 10$ The U.S. Food and Drug Administration withdrew the emergency authorization for hydroxychloroquine on June 15, 2020; however, several countries still continue to use hydroxychloroquine for COVID-19 treatment. ${ }^{11}$ 
Patients with comorbidities are vulnerable to infection and mortality due to COVID-19. Therefore, many patients with COVID-19 are exposed to a situation in which other drugs are concurrently administered with hydroxychloroquine. However, the risk of QT prolongation caused by DDIs between hydroxychloroquine and other co-medications has not yet been assessed comprehensively. The lack of evidence on the level of QT prolongation risk caused by DDIs challenges both physicians and regulators to select appropriate options for COVID-19 treatment.

To analyze the risk of QT prolongation caused by DDIs retrospectively from the perspective of real-world data, a large amount of electrocardiogram (ECG) results and drug prescription records are needed. Drug prescription data are usually easily accessible in electronic medical records (EMR), but extracting QTc interval information from the ECG results stored in hospital information systems could be a barrier to conducting large-scale studies with ECG data. We have made an effort to collect portable ECG results from inpatients and outpatients for the last few years. ${ }^{12}$ In this database, ECG parameters such as RR, PR, QRS, QT, and QTc intervals were extracted from raw ECG signals. The ECG database enabled us to conduct a study to provide direct evidence of QT prolongation caused by DDIs between hydroxychloroquine and other co-medications.

In this study, we aimed to evaluate the risk of QT prolongation caused by DDIs between hydroxychloroquine and other frequently used co-medications in real-world practice by conducting largescale retrospective case-control studies.

\section{Methods}

This retrospective study was conducted based on EMR data. The Institutional Review Board of Ajou University Hospital approved (IRB No. AJIRB-MED-MDB-19-406) and waived the requirement for informed consent because only anonymized data were used retrospectively. All research was performed in accordance with relevant guidelines and regulations.

\section{Data Sources}

We used the EMR database of Ajou University Hospital, a tertiary teaching hospital in Korea, recorded between January 1996 and May 2018. The database included 177,841,556 prescriptions, 379,994,144 laboratory test results, and 3,024,891 patient demographics.

QTC values from the same observational period were extracted from the local ECG repository in the MUSE $^{\mathrm{TM}}$ system. ${ }^{12,13}$ The ECG report typically contains both alphanumeric values and waveform graphs. The QTC data were extracted by parsing alphanumeric data from the PDF data extracted from the ECG repository using the web-scraping technique in our previous study. ${ }^{12}$ This ECG database contains 1,040,752 ECG results from 447,632 patients as of January 2018 (Figure 1). 


\section{Study design and population}

This study consisted of a series of retrospective case-control studies. In each study, we assessed the risk of QT prolongation due to DDI between hydroxychloroquine and one of the drugs (target drug) concomitantly used. We iterated this process for selected candidate drugs used in the subject hospital. The differences in drug use between patients with QT prolongation and those without QT prolongation were compared (Figure 1). QT prolongation was defined as an event where corrected QT (QTC), calculated using the Bazett formula, was greater than $450 \mathrm{~ms}$ for males and $460 \mathrm{~ms}$ for females.

We excluded the following ambiguous ECG results: (1) automatically duplicated ECG measurements known as systemic errors ( $n=84)$, and (2) sequentially measured ECG performed within 30 min, because these may reflect that the previous measurement could be an error $(n=47,700)$. We then randomly selected one ECG result per patient $(n=447,632)$. ECGs without sex or age information in the EMR and ECGs with age outliers (age $<0$ and age $>120)$ were removed $(n=3,057)$.

\section{Selection of candidate drugs for DDI risk analysis}

To select candidate drugs, we first extracted all drugs prescribed concomitantly with hydroxychloroquine in the window of interest (from 7 days prior to the date of ECG measurement) from the EMR. Then, drugs used concomitantly more than 10 times were included in the analysis. The list of selected drugs and their frequency of concurrent use are provided in Supplementary Table S1 online.

\section{Definition of covariates}

The following variables were included in the analysis to adjust for possible confounding factors on QT prolongation: (1) demographic information including sex and age at the ECG examination date, (2) comorbidities recorded in the EMR within a year before the ECG measurement date based on the International Classification Disease-10 (ICD-10). These comorbidities included myocardial infarction, congestive heart failure, ischemic stroke, hemorrhagic stroke, diabetes mellitus, hypothyroidism, renal disease, AIDS/HIV, alcohol abuse, drug abuse, liver disease, and severe liver disease; (3) the latest serum potassium and calcium levels within a year before the ECG measurement date. In patients without laboratory test results within 1 year before the ECG examination date, we imputed the missing values using the median value from patients of the same age group divided by 10-year intervals, and (4) the use of other drugs known to increase the risk of QT prolongation prescribed within 7 days before ECG measurement. The list of medications and comorbidities applied as parametric covariates is shown in Supplementary Table S2 online. 


\section{Subgroup analysis}

Because the risk of QT prolongation varies according to age and sex, we conducted a subgroup analysis. For this, we divided the subjects by sex (male and female) and age (age equal to or under 60 and age over 60 ). In this subgroup analysis, we included all drugs that were included in the main analysis.

\section{Statistical analysis}

We compared the demographic characteristics of the subjects, laboratory test results, comorbidities present, medication use, and year of the ECG measurement between the QT prolongation cases and controls using Pearson's chi-square tests (for categorical data) and independent two-sample t-tests (for continuous data). We iterated the process of fitting multiple logistic regressions, and in each iteration, one of the target drugs and an interaction term between hydroxychloroquine and the target drug were included (Figure 1). Using logistic regression models, we estimated the odds ratios (ORs) for each single drug and ORs for its concomitant use with hydroxychloroquine (interaction term) were calculated. In the current study, interaction term with a level of significance at $p<0.05$ was considered significant.

Data management was performed using Azure data studio version 1.19.0, and all statistical analyses were conducted using Python version 3.7 and the Python package Statsmodel version 0.11.1. The Python packages Matplotlib version 3.2.2, and seaborn Python packages version 0.10 .0 were also used for visualization of the data and results.

\section{Results}

\section{Baseline characteristics}

The total number of subjects included in the final analysis was 444,575 . Among them, the QT prolongation case group had 58,258 subjects and the control group had 386,317 subjects. Of the total patients, 218,997 were men and 225,578 were women. 1,417 patients received hydroxychloroquine within 7 days before their ECG examination date. The baseline characteristics of the patient group are summarized in Table 1.

A total of 118 medications were investigated, among which significant $(p<0.05)$ DDls were observed in the 12 medications. The results from each iteration with these 12 medications are shown in Table 2 . The results of the analysis of all medications are included in Supplementary Table S3 online. The full result table of tramadol is presented in Supplementary Table S4 online as an example.

\section{Multi-drug interaction analysis}


To analyze the DDIs of the 12 drugs with hydroxychloroquine, we created interaction plots. We eventually observed DDIs in all 11 drugs except tacrolimus in the direction of prolonging the QT interval (Figure 2).

\section{Subgroup analysis}

We divided the patient group into four subgroups according to sex and age. We then investigated whether the drugs used in each subgroup showed significant $(p<0.05)$ DDIs with hydroxychloroquine. We found that five drugs (tramadol, trimebutine, nifedipine, sulfasalazine, and rofecoxib) showed significant ( $p<$ $0.05)$ DDIs with hydroxychloroquine in the female subgroup, and three drugs (tacrolimus, paracetamol, and propacetamol) showed significant $(p<0.05)$ DDIs in the male patient group. Nine drugs (rosuvastatin, tacrolimus, sulfasalazine, trimebutine, ranitidine, ranitidine, diltiazem, celecoxib, paracetamol, and aceclofenac) showed DDIs with hydroxychloroquine in the group under 60 years of age. In the elderly group over 60 years old, no drug was significant $(p<0.05)$, but five drugs (clopidogrel, bisoprolol, furosemide, tramadol, and trimebutine) implied the possibility of a DDI $(p<0.1)$. The ten drugs with the highest probability of interaction for each subgroup are described in Table 3.

\section{Discussion}

Using EMRs obtained at a tertiary hospital, we investigated DDIs between hydroxychloroquine and 118 other drugs. We observed significant $(p<0.05)$ DDIs in 12 drugs. Among them, piperacillin/tazobactam, clarithromycin, and furosemide showed a risk of QT prolongation in individual treatment, and a DDI in the direction of increasing QT prolongation risk was also observed. However, for eight drugs (trimebutine, tramadol, rosuvastatin, cyclosporin, sulfasalazine, rofecoxib, diltiazem, and isoniazid), DDI was present in the direction of increasing the risk of QT prolongation, even though the QT prolongation risk of individual drugs was not significant $(p<0.05)$.

It is well known that hydroxychloroquine can cause QT prolongation. ${ }^{14,15}$ With the concern that DDIs between hydroxychloroquine and other drugs may exacerbate side effects such as this, several studies on the DDI of hydroxychloroquine have been investigated $14,16,17$. However, existing studies have selectively studied the DDI between hydroxychloroquine and some drugs that captured the attention of clinicians, such as immunosuppressants or antibiotics. ${ }^{16-19}$

In the case of patients with severe COVID-19, dozens of drugs are prescribed simultaneously with hydroxychloroquine, not only immunosuppressants or antibiotics. These drugs are medications that are taken regularly to treat chronic underlying diseases or are prescribed to relieve the patient's symptoms without specific indications. Most of these drugs are known to have minimal effects on QT prolongation when administered individually, but there is a risk of prolonging the QT interval indirectly through DDI with hydroxychloroquine. To overcome this problem, we conducted a DDI study not only on selected drugs but for all drugs that were prescribed concurrently with hydroxychloroquine in the chosen period. 
Consequently, the risk of prolonging the QT interval through DDI was observed in 11 drugs, even though the risk of prolonging QT in individual prescriptions was observed with only three drugs.

According to the therapeutic class of these drugs, three antibiotics (clarithromycin, piperacillin, and isoniazid) showed DDIs, and the mechanisms of these three antibiotics were also varied. Clarithromycin is a macrolide, such as azithromycin. Therefore, a DDI between clarithromycin and hydroxychloroquine suggests the possibility of a DDI in the combined therapy of azithromycin and hydroxychloroquine, which was suggested as a COVID-19 treatment. ${ }^{20}$ In this study, azithromycin was not included since there was not a sufficient number of patients who used the drug concurrently with hydroxychloroquine. DDI studies for azithromycin would be required later using a more massive clinical database so this can be investigated further.

Besides, other antibiotics can also be lethal in diseases such as COVID-19, which is deeply associated with respiratory disease. Piperacillin is a drug commonly prescribed in the hospitalization of patients in intensive care units (ICU) due to respiratory diseases such as pneumonia. ${ }^{21}$ Isoniazid is a drug that is used to treat tuberculosis, a disease that has a harmful effect on the lungs in the long term. As with COVID-19, tuberculosis is more common in developing countries. ${ }^{22}$ Therefore, if patients in developing countries have to treat tuberculosis and COVID-19 simultaneously, delicate QT interval monitoring is required.

Rofecoxib showed DDI with hydroxychloroquine in our main analysis, and several NSAIDs, such as celecoxib and paracetamol, showed interactions with hydroxychloroquine in the main analysis and in the young female subgroup analysis. Meanwhile, trimebutine, a spasmolytic drug commonly used for indigestion, showed DDI in the main analysis and in the young female subgroup analysis. These drugs are prescribed extensively and are often administered even in non-essential situations. Formerly, the side effects of these drugs are known to be minor. As a result, doctors are not cautious about these drugs, and they are often used as over-the-counter medicines. However, this study showed the possibility of DDI between these drugs and hydroxychloroquine, which could cause QT prolongation. Individually nondangerous drugs could cause drug side effects due to DDI in environments where large amounts of drugs are co-administered, such as COVID-19. Unnecessary routine prescriptions should be reduced, and appropriate alternative drugs should be selected.

Tramadol, one of the most commonly prescribed opioids, also showed DDI interaction with hydroxychloroquine. The risk of QT prolongation in opioids has already been reported. ${ }^{23}$ In particular, after COVID-19, it became accessible to purchase drugs on a non-face-to-face basis, and so opioid use disorder is rapidly increasing. ${ }^{24,25}$ If patients with opioid use disorder are infected with COVID-19, it would be essential to prevent fatal cardiotoxic adverse effects through precise QT monitoring.

In the subgroup analysis, the drugs that showed DDIs differed substantially by subgroup. Only four drugs showed significant $(p<0.05)$ interactions in two or more subgroups among the drugs analyzed. These results suggest that the interaction of hydroxychloroquine with other drugs may vary by age and sex. 
However, the difference in the drugs showing interactions among subgroups could be due to the difference in prescription patterns for each subgroup. In the future, studies that focus on specific subgroups would require additional statistical methods to compensate for these prescription patterns.

It is common to manage DDIs in clinical practice, but the mechanisms of DDIs are not adequately understood. One possible hypothesis for the interaction of drugs is through the activity of the CYP 450 enzyme. ${ }^{26}$ In the risk of QT prolongation interaction observed in this study, 7 out of 11 drugs had inhibitory effects on the metabolic pathway of hydroxychloroquine. CYP $4502 C 8$, and 3A4/5, especially clarithromycin and diltiazem, are known potent inhibitors of CYP $4503 \mathrm{~A}^{27}$. In addition, tramadol is also known to share the CYP 450 3A4 enzyme with hydroxychloroquine. ${ }^{28}$ These drugs may interfere with the metabolism of hydroxychloroquine, leading to QT prolongation by raising the hydroxychloroquine concentration to more than necessary.

This study has some limitations. First, it used a single institutional database. In the future, a multi-center study would be required to further generalize the results of this DDI study. Second, the cardiotoxic side effects of hydroxychloroquine are the main problems that arose during the COVID-19 situation ${ }^{29}$, but this study did not include COVID-19 patients due to their lack in number. This is due to our stringent inclusion criteria that only patients who were administered hydroxychloroquine and other drugs concurrently within 7 days before ECG measurement were included in the study.

\section{Conclusion}

The current study investigated interactions with hydroxychloroquine in 118 drugs using EMR data. Among them, DDls were found in 11 drugs that could increase the risk of QT prolongation. In eight drugs (trimebutine, tramadol, rosuvastatin, cyclosporin, sulfasalazine, rofecoxib, diltiazem, and isoniazid), DDI was present in the direction of increasing the risk of QT prolongation, even though the QT prolongation risk of the individual drugs alone were not significant $(p<0.05)$. In situations where hydroxychloroquine and a substantial number of other drugs are concurrently administered, as in COVID-19 patients, the adoption of alternative drugs should be thoroughly considered in the context of DDIs.

\section{Declarations}

\section{ACKNOWLEDGEMENT}

This research was supported by a grant (19182MFDS406) from the Ministry of Food and Drug Safety in 2020. This research was also supported by a government-wide R\&D Fund project for infectious disease research (GFID), Republic of Korea (grant number: HG18C0067).

\section{AUTHOR INFORMATION}




\section{Affiliations}

Department of Biomedical Informatics, Ajou University School of Medicine, Suwon, Gyeonggi-do, Republic of Korea

Byung Jin Choi, Yeryung Koo, Tae Young Kim, Bumhee Park, Dukyong Yoon ${ }^{1}$

Department of Pulmonology and Critical Care Medicine, Ajou University School of Medicine, Suwon, Gyeonggi-do, Republic of Korea

Wou Young Chung, Yun Jung Jung, Ji Eun Park

Department of Cardiology, Ajou University School of Medicine, Suwon, Gyeonggi-do, Republic of Korea Hong-Seok Lim

Office of Biostatistics, Medical Research Collaborating Center, Ajou Research Institute for Innovative Medicine, Ajou University Medical Center, Suwon, Gyeonggi-do, Republic of Korea

Bumhee Park

Department of Biomedical Sciences, Ajou University Graduate School of Medicine, Suwon, Gyeonggi-do, Republic of Korea

Dukyong Yoon

Corresponding author

Correspondence to Dukyong Yoon and Bumhee Park

\section{COMPETING INTERESTS}

The authors declare no competing interests. 


\section{SUPPLEMENTARY INFORMATION}

Supplementary information is available online.

\section{DATA AVAILABILITY}

The datasets generated during and/or analyzed during the current study are available from the corresponding author on reasonable request.

\section{References}

1. Administration USFaD. Who coronavirus disease (covid-19) dashboard. 2020

2. Colson P, Rolain J-M, Lagier J-C, Brouqui P, Raoult D. Chloroquine and hydroxychloroquine as available weapons to fight covid-19. Int J Antimicrob Agents. 2020;105932

3. Gao J, Tian Z, Yang X. Breakthrough: Chloroquine phosphate has shown apparent efficacy in treatment of covid-19 associated pneumonia in clinical studies. Bioscience trends. 2020

4. Mubagwa K. Cardiac effects and toxicity of chloroquine: A short update. International Journal of Antimicrobial Agents. 2020:106057-106057

5. Roden DM, Harrington RA, Poppas A, Russo AM. Considerations for drug interactions on qtc in exploratory covid-19 (coronavirus disease 2019) treatment. Circulation. 2020

6. Andreani J, Le Bideau M, Duflot I, Jardot P, Rolland C, Boxberger M, Wurtz N, Rolain J-M, Colson P, La Scola B. In vitro testing of combined hydroxychloroquine and azithromycin on sars-cov- 2 shows synergistic effect. Microbial pathogenesis. 2020:104228

7. Gautret P, Lagier J-C, Parola P, Meddeb L, Mailhe M, Doudier B, Courjon J, Giordanengo V, Vieira VE, Dupont HT. Hydroxychloroquine and azithromycin as a treatment of covid-19: Results of an openlabel non-randomized clinical trial. International journal of antimicrobial agents. 2020:105949

8. Molina JM, Delaugerre C, Le Goff J, Mela-Lima B, Ponscarme D, Goldwirt L, de Castro N. No evidence of rapid antiviral clearance or clinical benefit with the combination of hydroxychloroquine and azithromycin in patients with severe covid-19 infection. Med Mal Infect. 2020;50:30085-30088

9. Chorin E, Wadhwani L, Magnani S, Dai M, Shulman E, Nadeau-Routhier C, Knotts R, Bar-Cohen R, Kogan E, Barbhaiya C. Qt interval prolongation and torsade de pointes in patients with covid-19 treated with hydroxychloroquine/azithromycin. Heart rhythm. 2020

10. Mercuro NJ, Yen CF, Shim DJ, Maher TR, McCoy CM, Zimetbaum PJ, Gold HS. Risk of qt interval prolongation associated with use of hydroxychloroquine with or without concomitant azithromycin among hospitalized patients testing positive for coronavirus disease 2019 (covid-19). JAMA cardiology. 2020

11. U.S. Food and Drug Administration CfDEaR. Letter revoking eua for chloroquine phosphate and hydroxychloroquine sulfate. 2020 
12. Kim Y-G, Shin D, Park MY, Lee S, Jeon MS, Yoon D, Park RW. Ecg-view ii, a freely accessible electrocardiogram database. PloS one. 2017;12:e0176222

13. Chung D, Choi J, Jang J-H, Kim TY, Byun J, Park H, Lim H-S, Park RW, Yoon D. Construction of an electrocardiogram database including 12 lead waveforms. Healthcare informatics research. 2018;24:242-246

14. Ducharme J, Farinotti R. Clinical pharmacokinetics and metabolism of chloroquine. Clinical pharmacokinetics. 1996;31:257-274

15. Stas P, Faes D, Noyens P. Conduction disorder and qt prolongation secondary to long-term treatment with chloroquine. International journal of cardiology. 2008;127:e80-e82

16. Paterson DL, Singh N. Interactions between tacrolimus and antimicrobial agents. Clinical infectious diseases. 1997;25:1430-1440

17. Nwankwo JO, Garba MA, Chinje CE, Mgbojikwe LO, Emerole GO. Possible chloroquine-induced modification of n-acetylation of isoniazid and sulphadimidine in the rat. Biochemical pharmacology. 1990;40:654-659

18. Pukrittayakamee S, Tarning J, Jittamala P, Charunwatthana P, Lawpoolsri S, Lee SJ, Hanpithakpong W, Hanboonkunupakarn B, Day NP, Ashley EA. Pharmacokinetic interactions between primaquine and chloroquine. Antimicrobial agents and chemotherapy. 2014;58:3354-3359

19. Miller AK, Harrell E, Ye L, Baptiste-Brown S, Kleim JP, Ohrt C, Duparc S, Möhrle JJ, Webster A, Stinnett S. Pharmacokinetic interactions and safety evaluations of coadministered tafenoquine and chloroquine in healthy subjects. British journal of clinical pharmacology. 2013;76:858-867

20. Omura S. Macrolide antibiotics: Chemistry, biology, and practice. Elsevier; 2002.

21. Perry CM, Markham A. Piperacillin/tazobactam. Drugs. 1999;57:805-843

22. Saunders MJ, Evans CA. Covid-19, tuberculosis, and poverty: Preventing a perfect storm. 2020

23. Behzadi M, Joukar S, Beik A. Opioids and cardiac arrhythmia: A literature review. Medical principles and practice. 2018;27:401-414

24. Sun Y, Bao Y, Kosten T, Strang J, Shi J, Lu L. Challenges to opioid use disorders during covid-19. The American Journal on Addictions. 2020;29:174

25. Alexander GC, Stoller KB, Haffajee RL, Saloner B. An epidemic in the midst of a pandemic: Opioid use disorder and covid-19. 2020

26. Sinz M, Wallace G, Sahi J. Current industrial practices in assessing cyp450 enzyme induction: Preclinical and clinical. The AAPS journal. 2008;10:391-400

27. Mayhew BS, Jones DR, Hall SD. An in vitro model for predicting in vivo inhibition of cytochrome p450 $3 a 4$ by metabolic intermediate complex formation. Drug Metabolism and Disposition. 2000;28:10311037

28. Lassen D, Damkier P, Brøsen K. The pharmacogenetics of tramadol. Clinical pharmacokinetics. 2015;54:825-836 
29. Giudicessi JR, Noseworthy PA, Friedman PA, Ackerman MJ. Urgent guidance for navigating and circumventing the qtc-prolonging and torsadogenic potential of possible pharmacotherapies for coronavirus disease 19 (covid-19). Mayo Clinic Proceedings. 2020

\section{Tables}

Table 1. Baseline characteristics of the study group.

\begin{tabular}{|c|c|c|c|}
\hline & \multicolumn{2}{|c|}{ QT prolongation } & \multirow[b]{2}{*}{$p$-value } \\
\hline & Case & Control & \\
\hline Total & 58,258 & 386,317 & \multirow{4}{*}{$\begin{array}{l}<0.001 \\
<0.001\end{array}$} \\
\hline Hydroxychloroquine, n (\%) & $266(0.5)$ & $1151(0.3)$ & \\
\hline & & & \\
\hline Men, n (\%) & $29,932(51.4)$ & $189,065(48.9)$ & \\
\hline Women, n (\%) & $28,326(48.6)$ & $197,252(51.1)$ & \\
\hline Age, mean (SD) & $54.9(20.8)$ & $42.5(20.4)$ & \multirow{9}{*}{$\begin{array}{l}<0.001^{*} \\
<0.001\end{array}$} \\
\hline Age, $n(\%)$ & & & \\
\hline-29 & $102,645(26.6)$ & $6,996(12.0)$ & \\
\hline 30-39 & $68,163(17.6)$ & $5,417(9.3)$ & \\
\hline $40-49$ & $69,555(18.0)$ & $8,289(14.2)$ & \\
\hline $50-59$ & $59,192(15.3)$ & $10,355(17.8)$ & \\
\hline $60-69$ & $47,296(12.2)$ & $10,767(18.5)$ & \\
\hline 70-79 & $30,296(7.8)$ & $11,027(18.9)$ & \\
\hline-80 & $9170(2.4)$ & $5407(9.3)$ & \\
\hline \multicolumn{4}{|l|}{ Laboratory test result } \\
\hline Potassium, mean (SD) & $4.0(0.6)$ & $4.1(0.4)$ & $<0.001^{\star}$ \\
\hline Calcium, mean (SD) & $8.9(0.7)$ & $9.2(0.5)$ & $<0.001 x$ \\
\hline \multicolumn{4}{|l|}{ Comorbidity } \\
\hline Myocardial infarction, n (\%) & $1,733(3.0)$ & $3,519(0.9)$ & $<0.001$ \\
\hline Congestive heart failure, $\mathrm{n}(\%)$ & $1,795(3.1)$ & $2,397(0.6)$ & $<0.001$ \\
\hline Ischemic stroke, n (\%) & $2,462(4.2)$ & $6,366(1.6)$ & $<0.001$ \\
\hline Hemorrhagic stroke, n (\%) & $1,506(2.6)$ & $2,199(0.6)$ & $<0.001$ \\
\hline Diabetes mellitus, n (\%) & $3,341(5.7)$ & $1,2417(3.2)$ & $<0.001$ \\
\hline Hypothyroidism, n (\%) & $246(0.4)$ & $1,977(0.5)$ & 0.005 \\
\hline Renal disease, n (\%) & $2,172(3.7)$ & $3,022(0.8)$ & $<0.001$ \\
\hline AIDS/HIV, n (\%) & $27(0.0)$ & $125(0.0)$ & 0.114 \\
\hline Obesity, n (\%) & $94(0.2)$ & $2,285(0.6)$ & $<0.001$ \\
\hline Drug abuse, $\mathrm{n}(\%)$ & $194(0.3)$ & $390(0.1)$ & $<0.001$ \\
\hline Liver disease, n (\%) & 1,972 (3.4) & $2,703(0.7)$ & $<0.001$ \\
\hline \multicolumn{3}{|c|}{ Year of QTc diagnosis (ref. year 1995-1999) } & $<0.001$ \\
\hline $1995-1999$ & $28,246(7.3)$ & $1921(3.3)$ & \\
\hline $2000-2004$ & $69,420(18.0)$ & $5,172(8.9)$ & \\
\hline $2005-2009$ & $89,273(23.1)$ & $10,262(17.6)$ & \\
\hline 2010-2014 & $102,983(26.7)$ & $16,396(28.1)$ & \\
\hline 2015-2019 & $96,395(25.0)$ & $24,507(42.1)$ & \\
\hline
\end{tabular}

Table shows baseline characteristics comparison between a group with QT prolongation and a group without QT prolongation. * mark indicates that the p-value was calculated through the two-sided T-test. Other variables were compared using chi-square tests. 
Table 2. Odds ratio of single drug use and interaction with hydroxychloroquine from each multiple logistic regression

\begin{tabular}{lcccccc}
\hline & \multicolumn{3}{c}{ Single drug use } & \multicolumn{4}{c}{ Interaction with hydroxychloroquine } \\
& OR & $95 \% \mathrm{Cl}$ & $p$-value & OR & $95 \% \mathrm{Cl}$ & $p$-value \\
\hline Trimebutine & 0.84 & $(0.76-0.94)$ & $<0.001$ & 2.17 & $(1.33-3.53)$ & $<0.001$ \\
Tacrolimus & 0.75 & $(0.58-0.97)$ & 0.03 & 2.27 & $(1.31-3.93)$ & $<0.001$ \\
Tramadol & 0.98 & $(0.90-1.07)$ & 0.66 & 1.70 & $(1.24-2.34)$ & $<0.001$ \\
Rosuvastatin & 0.93 & $(0.86-1.01)$ & 0.07 & 2.80 & $(1.40-5.58)$ & $<0.001$ \\
Ciclosporin & 0.52 & $(0.38-0.70)$ & $<0.001$ & 8.06 & $(3.05-21.29)$ & $<0.001$ \\
Sulfasalazine & 0.81 & $(0.57-1.16)$ & 0.25 & 2.12 & $(1.19-3.80)$ & 0.01 \\
Rofecoxib & 0.35 & $(0.12-0.97)$ & 0.04 & 8.89 & $(1.56-50.7)$ & 0.01 \\
Diltiazem & 0.84 & $(0.77-0.90)$ & $<0.001$ & 3.15 & $(1.29-7.72)$ & 0.01 \\
Piperacillin & 1.34 & $(1.16-1.56)$ & $<0.001$ & 17.85 & $(2.10-151.94)$ & 0.01 \\
Isoniazid & 1.20 & $(0.98-1.47)$ & 0.08 & 3.89 & $(1.17-12.98)$ & 0.03 \\
Clarithromycin & 1.45 & $(1.26-1.68)$ & $<0.001$ & 3.17 & $(1.08-9.28)$ & 0.04 \\
Furosemide & 1.72 & $(1.63-1.82)$ & $<0.001$ & 2.03 & $(1.01-4.08)$ & 0.05 \\
\hline
\end{tabular}

Detailed analysis results of 12 drugs whose interaction term with hydroxychloroquine was statistically significant $(p>0.05)$ in linear regression. 'Single drug use' columns show the Odds ratio (OR), 95\% Confidence interval $(95 \% \mathrm{Cl})$, and $p$-value of the analyzed drug in the linear regression, and 'Interaction with hydroxychloroquine' columns show $\mathrm{OR}, 95 \% \mathrm{Cl}$, and the $p$-value of the interaction term with hydroxychloroquine. P-value was calculated through the two-sided T-test.

Table 3. Ten drugs with the lowest $p$-value of interaction terms for each subgroup. 


\begin{tabular}{|c|c|c|c|c|c|c|}
\hline & \multicolumn{3}{|c|}{ Single drug use } & \multicolumn{3}{|c|}{ Interaction with hydroxychloroquine } \\
\hline & OR & $95 \% \mathrm{Cl}$ & $p$-value & OR & $95 \% \mathrm{Cl}$ & $p$-value \\
\hline \multicolumn{7}{|l|}{ By age } \\
\hline \multicolumn{7}{|l|}{ Over 60} \\
\hline clopidogrel & 1.013 & $0.947-1.083$ & 0.708 & 0.42 & $0.175-1.009$ & 0.052 \\
\hline bisoprolol & 0.856 & $0.734-0.997$ & 0.046 & 0.127 & $0.015-1.055$ & 0.056 \\
\hline furosemide & 1.445 & $1.346-1.551$ & $<0.0001$ & 2.326 & $0.972-5.567$ & 0.058 \\
\hline tramadol & 1.004 & $0.81-1.245$ & 0.972 & 2.604 & $0.876-7.738$ & 0.085 \\
\hline trimebutine & 0.924 & $0.799-1.069$ & 0.288 & 1.754 & 0.903-3.406 & 0.097 \\
\hline cimetidine & 1.054 & $0.949-1.171$ & 0.322 & 0.287 & $0.064-1.283$ & 0.102 \\
\hline amlodipine & 0.94 & $0.867-1.02$ & 0.136 & 0.551 & $0.247-1.231$ & 0.146 \\
\hline chlorphenamine & 1.085 & $0.981-1.199$ & 0.112 & 0.398 & $0.115-1.38$ & 0.147 \\
\hline diltiazem & 0.813 & $0.739-0.894$ & $<0.0001$ & 2.169 & $0.73-6.45$ & 0.164 \\
\hline meloxicam & 0.805 & $0.589-1.1$ & 0.174 & 1.521 & $0.841-2.749$ & 0.165 \\
\hline \multicolumn{7}{|c|}{ Equal to or under 60} \\
\hline rosuvastatin & 0.864 & $0.755-0.989$ & 0.034 & 7.253 & $2.437-21.587$ & $<0.0001$ \\
\hline tramadol & 0.916 & $0.8-1.049$ & 0.203 & 2.506 & $1.587-3.959$ & $<0.0001$ \\
\hline sulfasalazine & 0.767 & $0.491-1.2$ & 0.245 & 3.451 & $1.632-7.296$ & 0.001 \\
\hline tacrolimus & 0.593 & $0.438-0.804$ & 0.001 & 3.52 & $1.709-7.25$ & 0.001 \\
\hline trimebutine & 0.774 & $0.654-0.916$ & 0.003 & 2.558 & $1.238-5.285$ & 0.011 \\
\hline ranitidine & 1.199 & $1.103-1.303$ & $<0.0001$ & 0.346 & $0.152-0.789$ & 0.012 \\
\hline diltiazem & 0.974 & $0.843-1.126$ & 0.721 & 5.506 & $1.295-23.404$ & 0.021 \\
\hline celecoxib & 0.75 & $0.525-1.072$ & 0.115 & 2.217 & $1.126-4.363$ & 0.021 \\
\hline paracetamol & 0.961 & $0.879-1.05$ & 0.375 & 2.148 & $1.095-4.215$ & 0.026 \\
\hline aceclofenac & 0.739 & $0.572-0.954$ & 0.02 & 2.146 & $1.071-4.299$ & 0.031 \\
\hline \multicolumn{7}{|l|}{ By sex } \\
\hline \multicolumn{7}{|l|}{ Female } \\
\hline tramadol & 0.926 & 0.819-1.046 & 0.217 & 1.809 & $1.254-2.612$ & 0.002 \\
\hline trimebutine & 0.837 & $0.72-0.973$ & 0.02 & 2.29 & $1.344-3.905$ & 0.002 \\
\hline nifedipine & 1.297 & $1.116-1.509$ & 0.001 & 2.828 & $1.252-6.387$ & 0.012 \\
\hline sulfasalazine & 0.801 & $0.477-1.345$ & 0.402 & 2.508 & $1.179-5.334$ & 0.017 \\
\hline celecoxib & 0.76 & $0.64-0.904$ & 0.002 & 1.716 & $1.08-2.728$ & 0.022 \\
\hline tacrolimus & 0.722 & $0.479-1.088$ & 0.12 & 1.899 & $0.937-3.849$ & 0.075 \\
\hline prednisolone & 0.696 & $0.596-0.813$ & $<0.0001$ & 1.512 & $0.911-2.51$ & 0.11 \\
\hline furosemide & 1.462 & $1.341-1.595$ & $<0.0001$ & 1.971 & $0.843-4.611$ & 0.117 \\
\hline aceclofenac & 0.687 & $0.538-0.876$ & 0.002 & 1.701 & $0.868-3.336$ & 0.122 \\
\hline meloxicam & 0.763 & $0.569-1.022$ & 0.07 & 1.357 & $0.847-2.174$ & 0.204 \\
\hline \multicolumn{7}{|l|}{ Male } \\
\hline tacrolimus & 0.769 & $0.551-1.073$ & 0.122 & 4.64 & $1.589-13.546$ & 0.005 \\
\hline paracetamol & 1.145 & $1.048-1.252$ & 0.003 & 4.139 & $1.375-12.456$ & 0.012 \\
\hline propacetamol & 1.239 & $1.117-1.375$ & $<0.0001$ & 7.962 & $1.585-40.01$ & 0.012 \\
\hline nifedipine & 1.361 & $1.185-1.564$ & $<0.0001$ & 0.208 & $0.044-0.974$ & 0.046 \\
\hline furosemide & 1.934 & $1.79-2.09$ & $<0.0001$ & 3.99 & $0.986-16.153$ & 0.052 \\
\hline sulglicotide & 0.814 & $0.574-1.155$ & 0.248 & 3.665 & $0.982-13.679$ & 0.053 \\
\hline atorvastatin & 0.794 & $0.719-0.877$ & $<0.0001$ & 2.919 & $0.953-8.942$ & 0.061 \\
\hline clopidogrel & 0.992 & $0.923-1.067$ & 0.831 & 0.368 & $0.09-1.505$ & 0.164 \\
\hline azathioprine & 0.702 & $0.375-1.315$ & 0.269 & 0.197 & 0.019-1.999 & 0.169 \\
\hline tramadol & 1.023 & $0.909-1.152$ & 0.704 & 1.569 & $0.817-3.015$ & 0.176 \\
\hline
\end{tabular}

This table shows the ten drugs with the lowest $p$-value of the interaction term with hydroxychloroquine in each subgroup. Each subgroup was divided into over 60 years old and under 60 years old or divided into 
male and female. 'Single drug use' columns show the Odds ratio (OR), 95\% Confidence interval ( $95 \% \mathrm{Cl})$, and $p$-value of the analyzed drug in the linear regression, and 'Interaction with hydroxychloroquine' columns show $\mathrm{OR}, 95 \% \mathrm{Cl}$, and the $p$-value of the interaction term with hydroxychloroquine. $p$-value was calculated through the two-sided T-test.

\section{Figures}

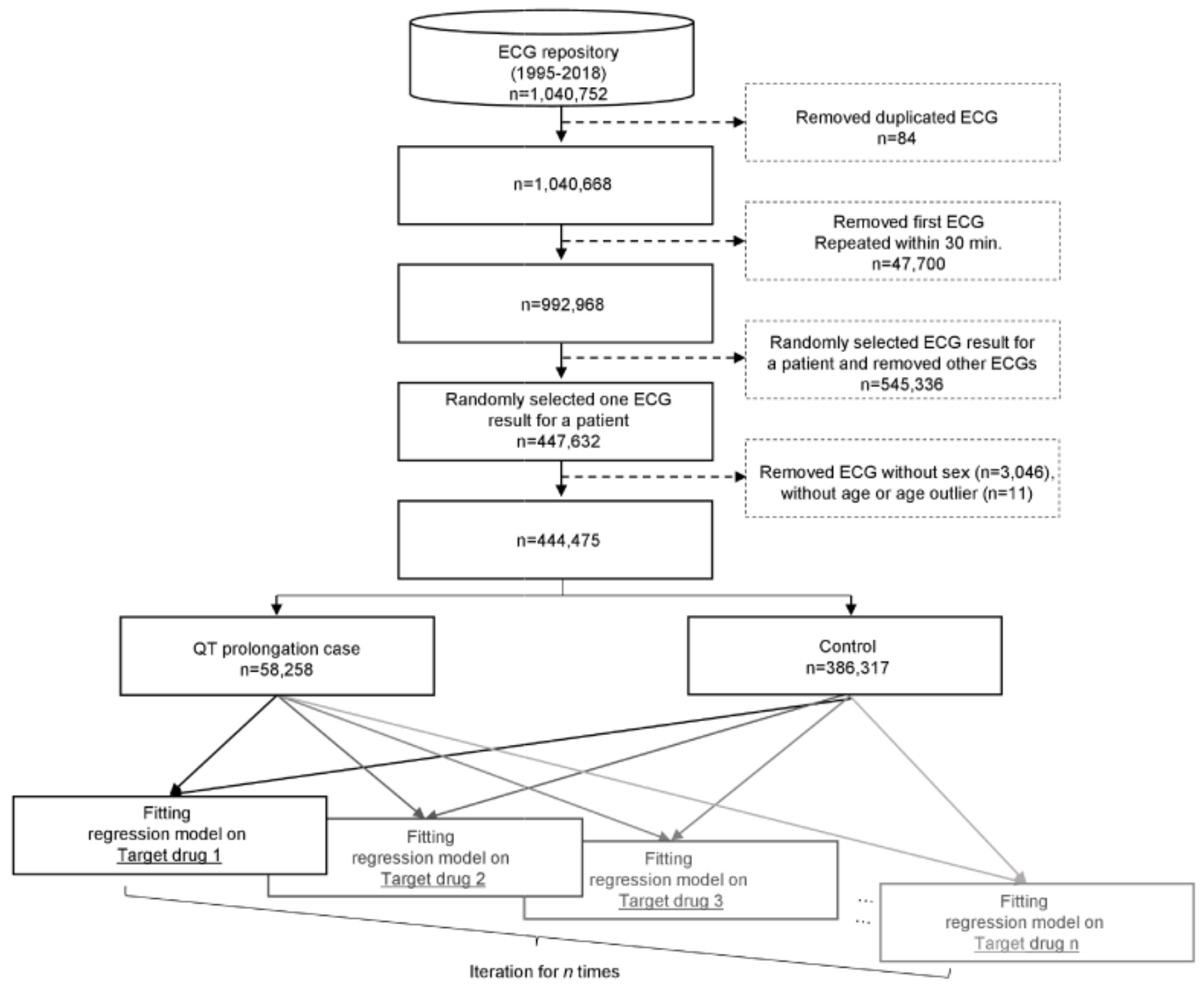

Figure 1

Flow chart of the study. Among the 1,040,702 ECG results, we removed automatically duplicated ECG results or repeated ECG results within 30 min, randomly selected one ECG result for a patient, and removed ECG results without sex and age information or outliers. A total of 444,575 ECGs were enrolled 
and divided into QT prolongation cases $(n=58,258)$ vs. control $(n=386,317)$. A regression model was iterated $\mathrm{n}$ times for each target drug.
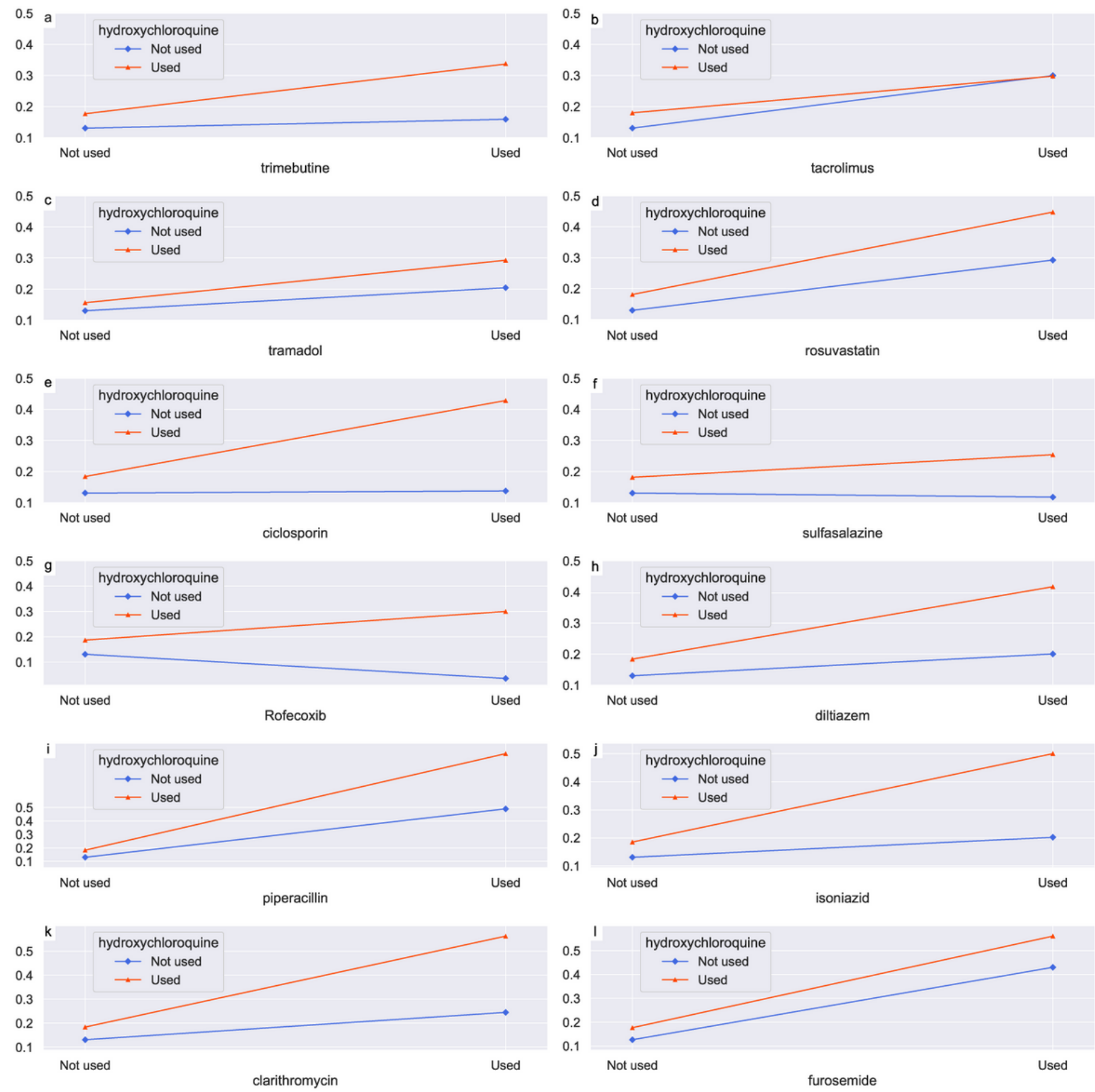

\section{Figure 2}

Interaction plots of 12 drugs with their observed interaction with hydroxychloroquine. The $x$-axis indicates the state of administration of the target drug while the y-axis represents the probability of QT prolongation when the patient takes the drug. The red line indicates concurrent use of target drug with hydroxychloroquine, the blue line represents the administration of the target drug alone. If there is no 
interaction, the two lines will be parallel. If the distance between the two lines is broader at the right side, the interaction exists in the direction of increasing the risk of QT prolongation, when it becomes narrow, the interaction exists in the opposite direction.

\section{Supplementary Files}

This is a list of supplementary files associated with this preprint. Click to download.

- supplementarysc.pdf 